\title{
1 An analysis of avian vocal performance at the note and song levels
}

3 David M. Logue ${ }^{1,2}$, Jacob A. Sheppard ${ }^{1}$, Bailey Walton ${ }^{1}$, Benjamin E. Brinkman ${ }^{3}$,

4 and Orlando J. Medina ${ }^{4}$

$6{ }^{1}$ Department of Psychology, University of Lethbridge. 4401 University Drive West, Lethbridge, 7 AB T1K3M4, Canada.

$8 \quad 2$ Departamento de Biología, Universidad de Puerto Rico. Mayagüez, PR 00682, USA

$9{ }^{3}$ Canadian Centre for Behavioural Neuroscience, University of Lethbridge. 4401 University

10 Drive West, Lethbridge, AB T1K3M4, Canada.

$11{ }^{4}$ Cabo Rojo National Wildlife Refuge. United States Fish and Wildlife Service. PO Box 510,

12 Boqueron, Cabo Rojo, PR 00622, USA

13 Corresponding author email: david.logue@uleth.ca

14

15 Short title: Song performance at two levels 


\section{Abstract}

Sexual displays that require extreme feats of physiological performance have the potential to reliably indicate the signaller's skill. The hypothesis that the structure of bird song is physiologically constrained remains controversial. We tested for evidence of performance constraints in Adelaide's warblers (Setophaga adelaidae) songs. At the note level, we identified three trade-offs with well-defined limits. At the song level, we identified two trade-offs, but their limits were less well-defined than the note-level limits. Trade-offs at both levels suggest that song structure is constrained by limits to the speed of both frequency modulation (while vocalizing and between notes) and respiration. Individual males experience the same trade-offs that characterize the population, but the intensity of those trade-offs varies among individuals. Performance metrics derived from the observed limits to performance varied moderately among individuals and strongly among song types. Note-level performance metrics were positively skewed, as predicted by the hypothesis that this population has experienced positive selection for constrained performance. We conclude that physiological limits on frequency modulation and respiration constrain song structure in male Adelaide's warblers. Further work is needed to determine whether receivers respond to natural levels of variation in performance, and whether performance correlates with singer quality. wood-warbler 


\section{Introduction}

39 Animal displays are dynamic signals that often function as sexual advertisements. Many sexual

40 displays challenge the signaller's motor abilities (Byers et al. 2010). For example, male

41 hummingbird courtship displays are pinnacles of length-specific velocity and acceleration

42 (Larimer and Dudley 1995; Clark 2009), male pronghorns' (Antilocapra americana) circle chase

43 displays require extreme angular acceleration (Byers 1997), and the success of male fiddler

44 crabs' (Uca perplexa) displays depends on the speed and height of their waving claws (Murai

45 and Backwell 2006). In these examples and others, it appears that female choice for high

46 performance has promoted the evolution of displays that reliably showcase males' abilities to

47 perform near their species' physiological limits (Smith and Harper 2003; Byers et al. 2010;

48 Bradbury and Vehrencamp 2011). Bird song is another sexual display that may fit that

49 description (Nowicki et al. 1992; Podos 1997; Podos et al. 2009).

\section{Performance constraints in bird song}

51 Song performance has been proposed to serve as a reliable signal of sender condition that is

52 salient to both male and female receivers (reviewed in Podos 2017). To sing, a bird must rapidly

53 coordinate the output of its brain, syrinx, bill, and respiratory system. Motor constraints on any

54 of these systems, or on the ability to coordinate them, could generate reliable correlations

55 between motor abilities and song structure, and signal receivers could learn about a signaller's

56 quality by listening to his or her song. A history of female choice for (or male deference to)

57 males that sing with high performance could explain the widespread evolution of songs with

58 rapid changes in fundamental frequency and rapid sequences of notes.

59 Several physiology studies indicate that motor constraints limit song structure (Hoese et al. 2000; 
60 Podos and Nowicki 2004; Plummer and Goller 2008). Most studies of motor performance in bird

61 song, however, do not directly measure physiological limits during song production (Cardoso

62 2017). Rather, they test the prediction that physiological constraints induce trade-offs between

63 acoustic traits. Podos (1997) performed the first such analysis when he described a trade-off

64 between the trill rate and frequency bandwidth in sparrow (Emberizidae) songs. Sparrow songs

65 with wide frequency bandwidth do not have high trill rates, and those with high trill rates do not

66 have wide frequency bandwidths (Fig. 5 in Podos 1997). This pattern can be at least partially

67 explained by limits on the speed of voiced frequency modulation (FM) and unvoiced FM

68 (frequency jumps between notes). Since Podos's pioneering work, several other studies have

69 identified trill rate vs. frequency bandwidth trade-offs in other species (reviewed in Wilson et al.

70 2014; Podos 2017).

71 Bird song researchers have identified several other acoustic trade-offs that suggest performance

72 constraints since Podos's studies of trill rate vs. frequency bandwidth trade-offs. A few have

73 started to study performance constraints at the level of the note. Relative to song-level analyses,

74 note-level analyses can be expected to provide clearer evidence of trade-offs if there is less

75 unmeasured structural variation among notes than there is among songs. For a given number of

76 recordings, note-level analysis also produces more data points than song-level analysis, resulting

77 in a more thorough description of acoustic space. Finally, note-level analyses permit tests of

78 hypotheses about specific physiological constraints, because they allow researchers to isolate

79 song elements related that might be subject to that constraint.

80 A recent note-level study showed that the difference in fundamental frequency between the end

81 of one note and the beginning of the next note trades off against the length of the silent gap

82 between notes (Geberzahn and Aubin 2014a). Larger frequency jumps between syllables require 
83 longer silent gaps. This trade-off suggests a limit to the speed of unvoiced FM, one of the

84 constraints that may contribute to the widely-observed trade-off between trill rate and frequency

85 bandwidth.

86 A second line of note-level research showed that longer notes are associated with longer silent

87 gaps before the subsequent note (Mota and Cardoso 2001; Cardoso et al. 2007). This trade-off

88 seems to be caused by a respiratory constraint. When singing rapidly, songbirds take unvoiced

89 mini-breaths between notes (Hartley and Suthers 1989). Long notes use more air than short

90 notes, so they require longer subsequent mini-breaths (Suthers and Zollinger 2004). Extremely

91 short notes are produced by a mechanism called 'pulsatile expiration,' which does not require

92 mini-breaths (Hartley and Suthers 1989).

\section{$93 \quad$ Measuring performance in bird song}

94 Trade-offs in acoustic traits can be used to quantify song performance. Cardoso (2017, p. e29)

95 defines song performance as 'the degree of challenge to the motor system, the respiratory

96 system, or other physiological processes involved in singing' (see also Byers et al. 2010). Song

97 performance metrics based on acoustic trade-offs estimate performance based on the acoustic

98 distance to an observed acoustic performance limit (Podos 2001). Vocalizations that are close to

99 or beyond the limit are high performance, whereas those that are far from the limit are low

100 performance (Fig. 1). Performance measurements derived in this way are called 'deviations'

101 because they measure the orthogonal deviation from the performance limit (Podos 2001).

\section{Critiques of bird song performance research}

103 Recently, research on bird song performance has come under criticism (Kroodsma 2017b, 
104 2017a). Kroodsma argues that performance is unlikely to be a reliable signal of singer quality

105 because it varies a great deal among song types, but little or not-at-all among individuals. One

106 way to test this hypothesis is to measure the repeatability (intraclass correlation coefficient, ICC)

107 of putative performance metrics for individuals and for song types (Cardoso et al. 2009). We

108 want to know whether a receiver's ability to compare individual singers' performance is limited

109 by the ICC for individuals, so our ICC calculation should reflect the receiver's assessment

110 strategy. The ICC for individuals should be based on the average performance of each song type

111 from each singer (as in Cardoso et al. 2009) if receivers first assess each song type separately,

112 and then average the performance of all of the song types in a singer's repertoire to estimate his

113 quality. However, if receivers assess singers' performance based on all of the songs they hear,

114 without first averaging within song type, the ICC should be based on all sampled songs. We did

115 not know how (or whether) receivers assess singers' performance, so we applied both methods.

116 Kroodsma (2017b) also argues that the bounded scatterplots that others have interpreted as

117 evidence of performance constraints (e.g., Figs. 1, 4, \& 5) do not represent performance limits,

118 but are a consequence of the cultural transmission of song structure. According to this

119 'constrained learning' hypothesis, it is physically possible for birds to sing beyond the observed

120 limits, but they do not because they have not learned songs beyond these limits. In any specific

121 case, the performance constraint hypothesis and constrained learning hypothesis are mutually

122 exclusive because they attempt to explain the same thing (bounded scatterplots). We used two

123 predictions to distinguish between these competing hypotheses. First, we predicted that

124 performance constraints would result in scatterplots with boundaries that slope in the expected

125 direction given plausible motor constraints. Constrained learning may or may not result in sloped

126 boundaries, and the direction of the slope should be random with respect to plausible motor 
127 constraints. Second, if a performance limit constrains the structure of vocalizations and singers

128 have evolved under positive selection for singing performance, vocalizations should cluster near

129 the limit, and deviation scores should skew away from the limit. The constrained learning

130 hypothesis generates a different prediction: Selection for species-specific song structure should

131 cause vocalizations to cluster in the middle of the distribution, where they are least likely to be

132 mistaken for heterospecific song. This pattern would produce a symmetrical deviation

133 distribution (skew $\approx 0$ ) with diffuse (low-density) edges.

\section{The present study}

135 We analysed a sample of male Adelaide's warbler (Setophaga adelaidae) songs for evidence of 136 performance constraints. We tested for three trade-offs at the note level and four trade-offs at the

137 whole song level, while controlling for variation attributable to individuals. We then analysed the

138 deviation scores. We tested whether receivers might be able to distinguish high-performance

139 singers from low-performance singers by estimating note-level and song-level ICC's for

140 individuals. We also estimated ICC's for song types, to better understand the relationship

141 between song type repertoires and vocal performance, and to permit comparisons with other

142 species. Finally, we tested opposing predictions of the constrained learning and constrained

143 performance hypotheses by measuring the skewness of the deviation distributions. Although

144 several previous studies have examined acoustic trade-offs in bird songs, this study is

145 distinguished by its comprehensiveness, sample size at the note level, and novel analytic

146 approach. 


\section{Methods}

\section{Study species}

150 Adelaide's warbler is a socially monogamous insectivore endemic to Puerto Rico and Vieques.

151 Mated pairs maintain all-purpose territories throughout the year (Toms 2010). Male songs are

152 frequency modulated trills (Fig. 2). Individual males sing repertoires of song types (avg. $\approx 23$

153 songs), many of which are shared with other males (Staicer 1991; Kaluthota et al. 2019). Each

154 male's repertoire comprises two categories, A and B, which are characterized by distinct times of

155 delivery, song rates, and song switching frequencies (Staicer 1991; Kaluthota et al. 2019). The

156 individual notes comprising songs are structurally simple, with almost all of the energy

157 concentrated in the fundamental frequency. There exists considerable among-note variation in

158 length, frequency, and frequency modulation (Fig. 2).

\section{Ethics}

160 This research was approved by the Institutional Animal Care and Use Committee at the

161 University of Puerto Rico, Mayagüez (17 September, 2010). It adheres to the ASAB/ABS

162 Guidelines for the use of animals in research. Birds were captured under D.M.L.'s federal bird

163 banding permit (no. 23696). The U.S. Fish and Wildlife Service granted permission to work at

164 the Cabo Rojo Wildlife Refuge (permit 2012-01).

\section{Recording and scoring}

166 We recorded nine mated male Adelaide's Warblers at the Cabo Rojo National Wildlife Refuge

167 (US Fish and Wildlife Service) during the breeding season between March and June, 2012. Each

168 male was recorded on four days, from 45 minutes before sunrise until 2 hours and 45 minutes

169 after sunrise. Consecutive recordings of a given male were separated by at least four days, except 
170 on two occasions when recordings were made on consecutive days because of logistical

171 constraints. Recordings were made with Marantz PMD 661 digital recorders and Sennheisser

172 ME67 shotgun microphones (file format = wav, sampling rate $=44.1 \mathrm{KHz}$, bit depth $=16$ bits).

173 This is the same set of recordings used in a previous study of short-term variation in song

174 performance (Schraft et al. 2017), a methods paper on song sequences (Hedley et al. 2018), and

175 an analysis of singing modes (Kaluthota et al. 2019).

176 We visualized recordings as sound spectrograms in Syrinx PC v.2.6 (J. Burt, Seattle, WA;

177 Settings: Blackman window, transform size $=1024$ points). Each song recording from a focal

178 male was assessed for recording quality. One person (D.M.L.) assigned song recordings to song

179 types. The inter-rater reliability of song type scoring of these recordings was estimated to be

$180100 \%$ within an individual bird, and $87 \%$ among individuals (Kaluthota et al. 2019). We only

181 used high-quality recordings (high signal-to-noise ratio, minimal overlap with other sounds) for

182 song measurements. High quality song recordings were analysed in Luscinia v2.14 (max. freq. =

$18310 \mathrm{kHz}$, frame length $=5 \mathrm{~ms}$, time $\mathrm{step}=1 \mathrm{~ms}$, dynamic range $=35 \mathrm{~dB}$, dynamic equalization $=$

$184100 \mathrm{~ms}$, de-reverberation $=100 \%$, de-reverberation range $=100 \mathrm{~ms}$, high pass threshold $=1.0 \mathrm{kHz}$,

185 noise removal $=10 \mathrm{~dB}$; Lachlan 2007). We loosely outlined the trace of each note with a stylus

186 on a touchscreen monitor, and Luscinia's algorithms identified the signal and rejected

187 background noise. Acoustic parameters for all notes were output to a spreadsheet. Luscinia offers

188 several frequency metrics. We chose peak frequency because visual inspection of spectrograms

189 showed that it tracked the fundamental frequency better than the fundamental frequency metric.

190 Analysis: trade-offs

191 The note-level analysis omitted the one or two low-amplitude notes that began some songs and 
192 the final note of all songs. We omitted final notes because it was impossible to define the length

193 of the silent gap following the last note. For the note-level analyses, we analysed the frequency

194 bandwidths and durations of both notes and silent gaps (Fig. 3). Following Cardoso (2013), our

195 measures of frequency bandwidth (BW) comprise the ratio of the maximum frequency to the

196 minimum frequency. Gap length and gap BW are taken from the silent gap after the focal note.

197 Gap BW is based on the end of the focal note and the beginning of the subsequent note. We

198 tested three comparisons at the note level that might reveal trade-offs indicative of performance

199 constraints: note length vs. gap length (respiratory), note BW vs. note length (voiced FM), and

200 gap BW vs. gap length (unvoiced FM).

201 We chose four parameters for the song-level analyses: trill rate, average frequency bandwidth,

202 percent of sound, and duration. Trill rate (TR) was calculated as the number of notes in the song

203 minus one, divided by the time from the beginning of the first note to the beginning of the last

204 note. We excluded the final note from this calculation because TRs based on the full song

205 necessarily omit the 'gap' after the last note, biasing estimates upward for songs with fewer

206 notes. Adelaide's warbler songs are frequency modulated trills (Fig. 2), so the total frequency

207 bandwidth of a song is only weakly related to the amount of FM in the song. We therefore

208 calculated a song's BW as the average BW of the notes in the song. Percent of sound (PoS) is the

209 percent of the song that is voiced. It was calculated as the sum of note lengths, divided by the

210 total length of the song, multiplied by 100. Lastly, we measured song length because many kinds

211 of performance increase in difficulty with increasing duration (Byers et al. 2010). We tested four

212 comparisons that might reveal trade-offs at the song level: TR vs. mean BW (FM, respiratory),

213 length vs. TR (respiratory endurance), length vs. PoS (respiratory endurance), and TR vs. PoS

214 (respiratory). 
215 Wilson et al. (2014) recommend quantile regression to test for acoustic trade-offs. Quantile

216 regression produces a linear function to estimate a defined quantile Y over a range of X (Cade

217 and Noon 2003). We conducted mixed quantile regression analyses to characterize potential

218 trade-offs while accounting for the non-independence of acoustic units from the same individual

219 (Geraci 2014). To the best of our knowledge, this is the first study to use mixed quantile

220 regression to test for acoustic trade-offs. To conform to recommended best practices, we treated

221 both intercepts and slopes as random variables (Barr et al. 2013). We predicted positive lower

222 boundaries for all note-level analyses, because higher values of acoustic variable X were

223 predicted to constrain the minimum values of variable Y (as in Geberzahn and Aubin 2014a). In

224 contrast, we predicted negative upper boundaries for all song-level analyses, because higher

225 values of variable $\mathrm{X}$ were predicted to constrain the maximum values of variable $\mathrm{Y}$ as in (as in

226 Podos 1997). For the note-level dataset, we tested lower boundaries with $10^{\text {th }}$ quantile

227 regressions $(\mathrm{tau}=0.10)$, following advice in Wilson et al. (2014). Similarly, we ran $90^{\text {th }}$ quantile

228 regressions $(\mathrm{tau}=0.90)$ to estimates upper boundaries in the song-level dataset (Wilson et al.

229 2014).

230 Population-level performance limits could arise from a pooled analysis of individuals that do not

231 themselves exhibit trade-offs. For example, some individuals might sing high-trill-rate, low-

232 bandwidth songs while others sing low-trill-rate, high-bandwidth songs, resulting in a sloping

233 limit to the population's distribution when individuals' data are pooled. Alternatively, different

234 individuals may be subject to similar trade-offs, which combine to produce a population-wide

235 trade-off. We therefore examined data from individual birds for evidence of trade-offs. We also

236 asked whether different individuals experience trade-offs differently by generating a reduced

237 model without random slopes and comparing its Akaike Information Criterion (AIC) value 
against that of the full model. Differences in AIC values ( $\triangle \mathrm{AIC})$ greater than 20 were interpreted

242 regression lines. To show individual variation near the boundaries, we also present zoomed-in

243 views of the boundary regions with separate colours for each individual and polygons that mark

244 the limit of each individual's distribution. Polygons were generated with the geom_encircle

245 command in the ggalt package (Rudis et al. 2017), with settings s_shape $=1$ (no added curvature)

246 and expand $=0$ (polygon edges intersect extreme points). Finally, we present separate

247 distributions for each individual in the electronic supplementary material.

\section{Analysis: Performance metrics}

249 Deviation scores were calculated as the orthogonal distance from the quantile regression lines,

250 such that higher (more positive) values indicate lower putative performance (Podos 2001). We

251 estimated intra-class correlations (ICCs) to test how repeatable individuals were with respect to

252 note-level deviation scores averaged over songs and song-level deviation scores. We conducted

253 ICCs for individuals using both the entire sample and the average scores for each song type

254 within individual (see Introduction). We also tested the repeatability of song types for note-level

255 deviation scores averaged over songs and song-level deviation scores. We used log-likelihood

256 tests to generate p-values for the ICCs.

257 We generated Pearson's correlation matrices of deviation scores for notes and songs. The song-

258 level correlation matrix included song-level deviations, note-level deviations averaged over

259 songs, and the performance metric Frequency Excursion (FEX). Frequency excursion attempts to 
260 estimate the speed with which the vocal apparatus adjusts frequency by measuring the rate of

261 change in the fundamental frequency, including during silent gaps (Podos et al. 2016; Schraft et

262 al. 2017). We calculated the skewness of the deviation scores to test competing predictions of the

263 constrained performance and constrained learning hypotheses.

264 All statistics were conducted in R Studio (Team 2015). Mixed quantile regressions relied on the

265 lqmm package (Geraci 2014). Intra-class correlations were assessed with the rptR package

266 (Stoffel et al. 2017). Data visualizations relied on the package ggplot2 (Wickham and Chang

267 2008). Data and $\mathrm{R}$ code are available at (DRYAD link when available).

269 Results

270 Note-level: Descriptive statistics

271 Subjects contributed an average of $320 \pm 185$ (average \pm SD) songs, belonging to $20.8 \pm 3.7$ song

272 types and comprising $7622 \pm 4568$ notes, to our analyses (Table 1 ). On average, notes were 50.8

$273 \pm 23.9 \mathrm{~ms}$ long and silent gaps were $35.7 \pm 11.5 \mathrm{~ms}$ long. Our measure of note-level frequency

274 bandwidth, the ratio of maximum peak frequency to minimum peak frequency, averaged $2.0 \pm$

2750.57 for notes, and $1.93 \pm 0.57$ for silent gaps.

\section{Note level: Trade-offs}

277 Mixed quantile regression analyses for note length vs. gap length (intercept $=12.87$, slope $=$

$\left.2780.24, \mathrm{p}_{\text {slope }}<0.0001\right)$ and note BW vs. note length (intercept $=18.45$, slope $=8.46, \mathrm{p}_{\text {slope }}<$

279 0.0001) were significant and positive, and all individuals' slope estimates were positive (Table 
ESM-1). The analysis of gap BW vs. gap length produced a positive slope estimate (intercept=

28123.02 , slope $\left.=3.19, \mathrm{p}_{\text {slope }}=0.03\right)$, but the $\mathrm{p}$-value was marginally significant at the $\alpha=0.05$

282 level, and eight out of nine individuals' slope estimates were positive. Individuals' slopes varied 283 significantly in the note length vs. gap length $(\triangle \mathrm{AIC}=981)$ and gap $\mathrm{BW}$ vs. gap length $(\Delta \mathrm{AIC}=$

284 2724) comparisons, but not in the note BW vs. note length comparison ( $\triangle \mathrm{AIC}=-178$ ).

285 Pooled scatterplots revealed sharply demarcated, approximately linear lower boundaries, except

286 for a prominent bulge in the lower left part of the boundary on the note length vs. gap length plot

287 (Fig. 4a-c). Average quantile regression lines approximated the slopes of boundaries of the

288 distributions, but their fit was imperfect (Fig. 4). The fit of the average quantile regression line

289 was particularly poor for the gap BW vs. gap length distribution (Fig. 4c). The polygons and

290 individual-level scatter plots showed positively sloping lower boundaries for all individuals, and

291 inter-individual variation in the slopes of the boundaries (Figs. 4d-f; ESM-1-3). The bulge in the

292 lower left portion of the note length vs. gap length comparison is apparent in most of the

293 individual-level plots (Fig. ESM-1).

\section{Note-level: Deviations}

295 When we used the entire sample of songs, all three note-level deviations averaged over songs

296 were significantly repeatable by individual, with moderate repeatability estimates (Table 2).

297 Using the average deviation scores for each song type from each individual resulted in lower

298 repeatability estimates, and rendered the note BW vs. note length deviation ICC non-significant

299 at the $\alpha=0.05$ level. All three deviation scores were significantly repeatable among song types,

300 with moderate to high repeatability estimates.

301 Deviation scores from the gap BW vs. gap length and note length vs. gap length comparisons 
were highly positively correlated $(r=0.874)$. There was a moderate correlation between gap BW vs. gap length and note BW vs. note length scores $(\mathrm{r}=0.574)$, and a weak correlation between note BW vs. note length and note length vs. gap length deviations $(r=0.112)$.

305 The distributions of deviation scores were all positively (right) skewed (Table 2). Note-level

306 deviation scores were strongly skewed, while note-level deviations averaged over songs

307 produced moderately skewed distributions. We graphed deviation against rank to identify

308 statistical outliers that fell below the regression lines $(n=72)$. Most of these outliers were

309 attributable to either reverberation or background noise that was accidentally treated as signal

310 when the songs were outlined in Luscinia, resulting in two notes being merged into one. We

311 excluded these outliers from graphs, but we did not exclude them from statistical analyses

312 because they comprise a small proportion of the overall dataset $(\sim 0.1 \%)$, and pruning only those

313 scoring errors that were outliers would bias the dataset.

\section{Song level: Descriptive statistics}

315 TRs averaged $11.64 \pm 1.58$ notes / second. Average mean frequency bandwidths were $2.05 \pm$ 316 0.23. The average PoS was $59.80 \pm 3.52 \%$. Songs were $2025.56 \pm 299.11 \mathrm{~ms}$ long, with an FEX 317 score of $66.39 \pm 11.45$, on average.

\section{Song level: Trade-offs}

319 Quantile regression analyses indicated the presence of statistically significant, negatively sloping 320 upper boundaries for all four song-level comparisons, although the TR vs. PoS slope was only 321 marginally significant $\left(\mathrm{TR}\right.$ vs. mean BW: intercept $=3.19$, slope $=-0.074, \mathrm{p}_{\text {slope }}<0.0001$; length

322 vs. TR: intercept $=14.95$, slope $=-0.0011$, pslope $=0.0071 ;$ length vs. PoS: intercept $=70.38$, 
slope $=-0.003, \mathrm{p}_{\text {slope }}=0.0001 ;$ TR vs. PoS: intercept $=74.21$, slope $=-0.92$, plope $\left._{\text {slo }}=0.0356\right)$. All

324 of the individual slope estimates were negative except for one individual's length vs. TR

325 estimate (Table ESM-2). Individuals' slopes varied significantly in the TR vs. mean BW ( $\triangle \mathrm{AIC}$

$326=3529)$, length vs. TR $(\Delta \mathrm{AIC}=4648)$, and TR vs. $\mathrm{PoS}(\Delta \mathrm{AIC}=8473)$ comparisons, but not in

327 the length vs. PoS comparison $(\triangle \mathrm{AIC}=-23,945)$.

328 The pooled scatterplots suggest overall negative trends and loosely-defined negatively sloping

329 upper limits for the TR vs. mean BW and TR vs. PoS comparisons (Fig. 5a, g). The average $90^{\text {th }}$

330 quantile regression lines appeared to fit the upper boundaries of the TR vs. mean BW and TR vs.

331 PoS distributions well (Fig. 5a, g). Polygons and individual-level scatterplots from most

332 individuals showed evidence of negative relationships for the TR vs. mean BW and TR vs. PoS

333 comparisons (Figs. 5b, h, ESM-4, ESM-7). In contrast, the length vs. TR and length vs. PoS

334 pooled scatterplots, polygons, and individual-level scatterplots did not provide evidence of

335 negative relationships (Fig. 5c-f, ESM-5, ESM-6).

\section{Song level: Deviations}

337 All four song-level deviation scores and FEX were moderately repeatable among individuals

338 when all songs were considered (Table 3). When we used the average of each song type within

339 individual to calculate ICCs, all repeatability estimates were lower except for those derived from

340 the TR vs. PoS deviations, and the TR vs. mean BW repeatability was not statistically

341 distinguishable from zero. The song-level metrics of performance were moderately to highly

342 repeatable among song types.

343 FEX, song-level deviations, and note-level deviations averaged over songs were intercorrelated

344 (Table 4). We found strong negative relationships between FEX and each of the following 
345 deviation scores: TR vs. mean BW, mean note BW vs. note length and mean gap BW vs. gap

346 length. Rapid FM and high TRs are indicated by high FEX values and low deviations, so these

347 negative correlations may imply redundancy. By contrast, FEX was only weakly correlated with

348 the TR vs. PoS and mean note length vs. gap length deviations. The song level metric TR vs. PoS

349 deviation correlated strongly with the mean note-level deviations from the note length vs. gap

350 length and gap BW vs. gap length deviations. The mean gap BW vs. gap length deviations were

351 also highly correlated with the mean note length vs. gap length deviations and the mean note BW

352 vs. note length deviations, but those two were not strongly correlated with each other.

\section{Discussion}

355 Sexual displays can reliably indicate the signaller's skill when physiology constrains display

356 structure. We found evidence that the structure of a bird's song is constrained by the speed with

357 which singers can modulate frequency and replenish expired air. Some males consistently

358 performed closer to the estimated population-wide performance limits than others, so signal

359 receivers (e.g., potential mates and rivals) may be able to assess among-individual variation in

360 song performance. Among song type variation in performance metrics indicates that some song

361 types are more challenging to sing than others.

\section{Trade-offs and performance constraints}

363 We found strong evidence of three acoustic trade-offs at the note level. The note-level

364 scatterplots with sharply-defined, positively sloping boundaries comprise what we believe to be

365 the most compelling acoustic evidence of performance constraints in bird song to date (Fig. 4,

366 ESM-1-3). Individuals' scatterplots and slope estimates showed that each male was subject to the 
367 trade-offs that constrain vocal production. Inter-individual variation in slope estimates for the 368 note length vs. gap length and gap BW vs. gap length comparisons suggests the novel hypothesis

369 that high quality individuals are subject to weaker trade-offs (lower slopes) than are low quality

370 individuals.

371 The observed trade-off between note length and gap length supports the hypothesis that

372 respiratory performance constrains song structure (Figs. 4a, d). Relative to short notes, long

373 notes require birds to expire more air, necessitating longer subsequent mini-breaths to replenish

374 the bird's air supply in preparation for the next note. A similar pattern was previously identified

375 in male serin (Serinus serinus) and dark-eyed junco (Junco hyemalis) songs (Mota and Cardoso

376 2001; Cardoso et al. 2007). The lower boundary for the note length vs. gap length distribution

377 bulges downward at short note lengths in the pooled plot and in many of the individual plots

378 (Figs. 4a, ESM-1). We hypothesize that this bulge represents notes that are short enough for

379 birds to sing with pulsatile expiration (Hartley and Suthers 1989; Mota and Cardoso 2001). We

380 do not yet know whether Adelaide's warbler receivers respond to variation in respiratory

381 performance, but there is evidence that other species do. Male skylarks (Alauda arvensis)

382 increase the sound density of their songs when challenged with playback (Geberzahn and Aubin

383 2014b). Male dusky warblers (Phylloscopus fuscatus) that sing with consistently high amplitude

384 live longer and enjoy extra-pair paternity benefits (Forstmeier et al. 2002). Similarly, male zebra

385 finches (Taeniopygia guttata) that sing with higher sound density are preferred by females

386 (Holveck and Riebel 2007).

387 We interpret the results from the note BW vs. note length and gap BW vs. gap length

388 comparisons as evidence that frequency modulation speed is constrained (Figs. 4b, c, e, f). At the

389 limit of performance, large frequency changes require more time than do small frequency 
changes. Podos (1997) first described a constraint on the speed of FM in general, and Geberzahn and Aubin (2014a) identified the constraint on unvoiced FM in skylarks. We believe that the

392 present study is the first to specifically characterize a constraint on voiced FM. The apparent

393 physiological basis of both constraints is that the magnitude of FM scales with the magnitude of

394 physical change in the vocal apparatus and the vocal apparatus requires time to reconfigure itself

395 (Suthers 2004). The speed of FM may be constrained by the bird's ability to coordinate the

396 various component of the vocal apparatus (brain, left and right syrinx, upper vocal tract, etc.;

397 reviewed in Podos et al. 2009) that are involved in frequency modulation. Alternatively, a

398 limitation on any component could limit the entire system. For example, bill size constrains

399 singing speed in various taxa (Westneat et al. 1993; Podos 2001; Derryberry et al. 2012). There

400 is widespread evidence that signal receivers attend to variation in FM speed (Drăgănoiu et al.

401 2002; Ballentine 2004; Illes et al. 2006; Cramer and Price 2007; DuBois et al. 2009; DuBois et

402 al. 2011; Moseley et al. 2013; Vehrencamp et al. 2013; Phillips and Derryberry 2017a, 2017b).

403 We also found evidence of constrained performance at the song level. All four song-level

404 quantile regressions were statistically significant, but the visual data from the length vs. TR and

405 length vs. PoS comparisons were not compelling (Figs. 5c, d, e, f). We conclude that there is

406 insufficient evidence to support the hypotheses that song length (or the combination of length

407 and other variables) advertises singers' motor abilities. Singing does not require much more

408 oxygen than resting, and mini-breaths between notes may permit birds to escape respiratory

409 constraints on song length (Oberweger and Goller 2001; Suthers and Zollinger 2004). Another

410 study that found no evidence of constrained song length did find that birds sang longer songs

411 when they were vocally interacting with other males (Cardoso et al. 2009). The authors

412 concluded that 'the length of songs is a plastic trait that varies with social context in a way that 
413 seems to reflect overall motivation or singing intensity' (p. 904). A similar dynamic may be at

414 play in our study system.

415 We found robust evidence that TR trades off against mean BW, replicating Podos's (1997)

416 canonical finding in sparrows (see Podos et al. 2016 for a general review), as well as a previous

417 finding in other taxa (Wilson et al. 2014), including wood-warblers (Cardoso and Hu 2011). We

418 originally believed that the TR vs. mean BW trade-off was caused by constraints on FM speed

419 during voiced notes and unvoiced gaps. However, the modest correlations between TR vs. mean

420 BW deviations and the mean deviations from note BW vs. note length $(r=0.223)$ and gap BW

421 vs. gap length $(\mathrm{r}=0.310)$ suggest that note-level FM constraints may not fully explain the TR vs.

422 mean BW constraint. We conclude that TR vs. BW trade-offs probably emerge from some

423 combination of constraints to voiced FM, unvoiced FM, and note repetition rate. The TR vs. PoS

424 analysis provides further evidence that note repetition rate is constrained.

425 We tentatively conclude that the balance of evidence supports the existence of a TR vs. PoS

426 trade-off, such that fast trills include more silence than do slow trills. The regression results were

427 marginally significant $(\mathrm{p}=0.0356)$, and visual analysis indicated negative trends in the pooled

428 data, and some evidence of negative trends in the individual data (Figs. 5g, h, ESM-7). Although

429 other studies have examined metrics similar to PoS (Forstmeier et al. 2002; Leadbeater et al.

430 2005; Holveck and Riebel 2007; Cardoso et al. 2009), we believe that this is the first study to

431 show a relationship between PoS and TR. The physiological basis of this trade-off is probably

432 similar to the respiratory constraint responsible for the note length vs. gap length trade-off.

433 Indeed, the deviation scores from these two analyses are highly correlated $(r=0.886)$. To

434 approach the upper boundary of the TR vs. PoS distribution, a bird must both minimize gap

435 lengths, as in the note length vs. gap length trade-off, and also emit notes at a high rate. 
436 At the limit of performance, the relationship between acoustic variables varied among

437 individuals in both the TR vs. mean BW and TR vs. PoS comparisons. As in the note-level

438 analysis, we propose that some individuals may be more robust to trade-offs than others, and that

439 this variation may indicate quality.

\section{$440 \quad$ Comparing levels of analysis}

441 The evidence for trade-offs at the note level was much stronger than the evidence at the song

442 level. The boundaries of the note-level distributions were clearly defined, allowing us to see the

443 shape of the boundary (e.g., the node in Fig. 4a). In contrast, the song-level boundaries were

444 diffuse (compare Figs. 4 \& 5). We believe that the main cause of this difference is that notes

445 have a simpler structure than the songs they comprise. This simpler structure means that

446 measured acoustic variables describe notes more completely than songs. Unmeasured variation

447 among acoustic units contributes random error, making it harder to identify performance limits

448 with song-level variables. Notes are also more numerous than songs, and large sample sizes

449 permit more precise characterization of performance limits. It is often, but not always, possible

450 to scale up from note-level performance to song-level performance (see the discussion of TR vs.

451 PoS deviations above). We conclude that note-level analysis is a powerful approach for studying 452 acoustic trade-offs, but it cannot completely replace song-level analyses.

\section{Methodological considerations}

454 The present study revealed two important limitations to quantile regression. First, the line that it 455 produces is not always parallel to the border of the point cloud, reducing our ability to accurately

456 estimate performance limits and deviation scores. Part of the reason for the poor fit is that the

457 quantile regression algorithm is influenced by points that are distant from the focal edge. This 
effect is most apparent in the gap BW vs. gap length scatterplot (Fig. 4c), in which the large

459 number or long, low BW gaps influences the line to slope less steeply than the lower boundary.

460 When the regression line does not parallel the edge of the distribution, deviation scores

461 incorrectly weight their constituent variables. We overlooked this issue in the current study

462 because our lines fit well enough to demonstrate trade-offs and approximate deviation scores,

463 and because we did not want to introduce and justify a novel edge detection paradigm in a report

464 that already contained many analyses. A second problem with the quantile regression analyses is

465 that we found statistically significant quantile regressions even when plots did not appear to

466 show strong evidence of performance limits (compare Figs. 5c-5f to the results of the

467 corresponding quantile regressions). This problem was not unique to the lqmm package -

468 standard quantile regressions were also statistically significant (unpublished analyses).

469 Considering these limitations, we recommend that users supplement the results of quantile

470 regression analyses with visual inspection of scatterplots and encourage the continued

471 development of statistical methods for detecting and defining performance limits.

472 Our sample comprised many songs $(n=2879)$ and very many notes $(n=68,602)$, recorded from

473 relatively few individuals $(\mathrm{n}=9)$. This sampling scheme allowed us to characterize individuals'

474 distributions with high precision, especially at the note level. We were thus able to determine

475 whether individuals were subject to trade-offs, and whether constraints varied among

476 individuals. The large sample size of notes also resulted in dense point clouds that were

477 amenable to visual assessment. Thus, we had a great deal of power to establish within individual

478 patterns. Although the sample of individuals was modest, it was sufficient to test for population-

479 level trade-offs without pseudoreplicating individuals because all individuals were subject to

480 these note-level trade-offs. 


\section{Repeatability of performance metrics}

482 Most note-level deviation scores were repeatable by individual and by song type. ICC's for

483 individuals based on all songs indicated that $14-20 \%$ of variation in song performance can be

484 attributed to individual differences. Whether receivers can differentiate among singers'

485 performance is an empirical question that is amenable to experimental investigation. Simulation

486 models in which receivers sample varying numbers of songs from two singers and assess their

487 relative performance, would also be useful. Estimates of repeatability by individual based on all

488 songs tended to be considerably higher than those based on the averages for each song type

489 within individual (Table 2). This difference has implications for both signallers and receivers.

490 Signallers do not sing all song types in their repertoires with equal frequency, but instead sing

491 some song types more than others. Relative to a flat distribution of song types, the observed

492 distribution of song types resulted in greater individual distinctiveness. This finding suggests the

493 hypothesis that more skilled singers are able to sing demanding song types more often than are

494 less skilled singers. Turning to receivers, our results indicate that estimating the average

495 performance of all sampled songs would be a more efficient assessment strategy than would

496 averaging for each song type and then averaging over song types. The former strategy is also less

497 cognitively demanding because it includes fewer steps and requires less memory. Receivers'

498 assessment strategies require further investigation (Guilford and Dawkins 1991; Bateson and

499 Healy 2005; Podos 2017).

500 Performance metrics were moderately to highly repeatable among song types (Tables $2 \& 3$ ). If

501 deviation scores represent performance, then song types vary with respect to their performance

502 demands. The deviation scores that measured FM speed (those that included BW and FEX) were 
503 highly repeatable by song type. That result was unsurprising because we used patterns of

504 frequency modulation to classify song types (Fig. 2).

505 A previous study of male dark-eyed junco songs also found that repeatability estimates for song

506 types were higher than repeatability estimates for individuals (Cardoso et al. 2009). That study

507 averaged performance over song types within individuals before estimating individual ICC, and

508 arrived at similar estimates to the comparable analysis in our study. Their estimates of song type

509 repeatability were higher than ours, but that difference could be attributable to differences in

510 song type scoring. The authors conclude that receivers could use acoustic performance to

511 estimate singers' quality, but go on to write, 'the main conclusion from our results it that,

512 because most of the variation in performance depends on the song type, a receiver that compares

513 a few song types from different males is likely to obtain little information about performance

514 differences between males' (p. 905). In our study system, receivers would get considerably more

515 information if they based their assessments on all songs (rather than averaging performance

516 within song types and then within males). If high quality males sing more challenging song

517 types, averaging performance within song type before calculating repeatability would

518 underestimate receivers' abilities to discern singers' quality. Further, it is unlikely that

519 Adelaide's warbler receivers would only hear one or a few song types, because males rapidly

520 cycle through their song types during dawn singing (Staicer 1991; Kaluthota et al. 2019).

\section{Correlations among performance metrics}

522 Several pairs of performance metrics were positively correlated, suggesting that metrics are

523 partially redundant or that different kinds of performance covary positively. It appears that note

524 length vs. gap length deviation scores and gap BW vs. gap length deviation scores are highly 
correlated because short gaps generate low deviation scores in both comparisons. The correlation

526

527

528

529

530

531

532

533

534

535

coefficient is inflated by the poor fit of the quantile regression line in the gap BW vs. gap length

comparison (see above). Nevertheless, short gap lengths may indicate high quality with respect

to both FM speed and respiratory performance. The high correlation between note BW vs. note

length deviation and gap BW vs. gap length deviations may arise because short notes tend to be

followed by short gaps, and vice-versa (as shown in the note length vs. gap length comparison).

At the level of the whole song, we found that FEX correlated strongly with various performance

metrics that measure FM speed, but FEX was not strongly correlated with two metrics that seem

to measure respiratory performance. Thus, FEX is useful for measuring overall FM speed, and

note-level deviation scores are useful for parsing FM speed between voiced and unvoiced

portions of the song, but FEX does not measure respiratory performance. The strong correlation

between TR vs. PoS deviations and the deviation scores that involve gap length emphasizes the

importance of short inter-note intervals for various performance metrics. Overall, we did not find

evidence that FM performance trades off against respiratory performance.

\section{Skewness of performance metrics}

Note-level deviation scores were positively skewed, as predicted by the hypothesis that the

population has evolved under selection to sing near the physiological limits of performance

(Table 2). We did not find this pattern with the song-level deviations (Table 3). The difference

between patterns of skewness at the two levels of analysis may be attributable to the greater

statistical noise in the song-level metrics. We tentatively conclude that the note-level skewness

results support the performance constraint hypothesis and fail to support the constrained learning

hypothesis, but equivalent data from other study systems are needed. 


\section{Conclusions and future directions}

548 Most research on performance constraints in bird song is based on acoustic trade-off analysis,

549 but this paradigm has recently come under strong criticism (Kroodsma 2017b, 2017a). We

550 responded to these criticisms with a study that included multiple comparisons at two levels, a

551 note-level sample size that was nearly ten times larger than the largest comparable dataset

552 (Geberzahn and Aubin 2014a), and novel statistical approaches to control for pseudoreplication

553 and test for evidence of selection for high performance. We believe that this approach has

554 produced the most compelling acoustic evidence yet that physiological limits constrain the

555 structure of bird song.

556 We conclude that performance constraints on the speed of voiced FM, unvoiced FM, and

557 respiration limit the acoustic structure of male Adelaide's warbler songs. We hypothesize that

558 this species sings trills of rapid frequency sweeps because of a history of sexual selection for FM

559 and respiratory performance.

560 This study opens the door for future research on performance constraints in Adelaide's warbler

561 and other species. One critical question is whether male or female receivers attend to variation in

562 one or more performance metrics in this population. It would be particularly interesting to know

563 if they can accurately assess synthesized 'singers' with realistic performance ICC's. Future

564 studies should also examine the link between song performance and male phenotype,

565 performance and among-individual variation in song type use, and how performance varies

566 across contexts, including vocal interactions (Logue and Forstmeier 2008). 


\section{Acknowledgements}

568 We would like to thank the people who helped us obtain and process recordings: Daniel Pereira and

569 Josiris Rodriguez helped record birds; Paloma Sanchez-Jaureguí, Fabio L. Tarazona, Jorge Illanas,

570 Krystal Medina, and Alfredo Lamela helped score recordings; Tony Shlakoff, Casandra Logue, Joshua

571 Baez, Ruben Irizarry, and Alicia García processed song recordings in Luscinia; and Jesse McClure

572 measured FEX. We also thank Dr. Robert Lachlan for his advice and technical support on Luscinia.

573 Earlier versions of this manuscript were improved by comments from Hannes Schraft, Samantha Krause,

574 Nolan Gooding, Todd Freeburg and two anonymous referees. This work was supported by a Discovery

575 Grant from the Natural Sciences and Engineering Research Council of Canada (RGPIN-2015-06553) to

576 D.M.L., and two NSERC undergraduate summer research assistantships to J.S. and B.W.

\section{Declaration}

579 The authors have no conflicts of interest to declare. 


\section{References}

581 Ballentine B. 2004. Vocal performance influences female response to male bird song: an experimental test. Behavioral Ecology. 15(1):163-168.

Barr DJ, Levy R, Scheepers C, Tily HJ. 2013. Random effects structure for confirmatory hypothesis testing: Keep it maximal. Journal of Memory and Language. 68(3):255-278.

Bateson M, Healy SD. 2005. Comparative evaluation and its implications for mate choice. Trends in Ecology and Evolution. 20(12):659-664.

Bradbury J, Vehrencamp SL. 2011. Principles of Animal Communication: Second edition. Sunderland, Massachusetts: Sinauer.

Burnham KP, Anderson DR, Huyvaert KP. 2010. AIC model selection and multimodel inference in behavioral ecology: some background, observations, and comparisons. Behav Ecol Sociobiol. 65(1):23-35.

Byers J. 1997. American pronghorn: social adaptations and the ghosts of predators past. University of Chicago Press.

Byers J, Hebets E, Podos J. 2010. Female mate choice based upon male motor performance. Animal Behaviour. 79(4):771-778.

Cade BS, Noon BR. 2003. A gentle introduction to quantile regression for ecologists. Frontiers in Ecology and the Environment. 1(8):412-420.

Cardoso GC. 2013. Using frequency ratios to study vocal communication. Animal behaviour. 85(6):1529-1532.

600 Cardoso GC. 2017. Advancing the inference of performance in birdsong. Animal Behaviour. 125:e29-e32.

602 Cardoso GC, Atwell JW, Ketterson ED, Price TD. 2007. Inferring performance in the songs of dark-eyed juncos (Junco hyemalis). Behavioral Ecology. 18(6):1051-1057.

Cardoso GC, Atwell JW, Ketterson ED, Price TD. 2009. Song types, song performance, and the use of repertoires in dark-eyed juncos (Junco hyemalis). Behavioral Ecology. 20(4):901-

607 Cardoso GC, Hu Y. 2011. Birdsong performance and the evolution of simple (rather than

608 elaborate) sexual signals. The American naturalist. 178(5):679-686.

609 Clark CJ. 2009. Courtship dives of Anna's hummingbird offer insights into flight performance

610 limits. Proceedings of the Royal Society B: Biological Sciences. 276(1670):3047-3052. 
Cramer ERA, Price JJ. 2007. Red-winged blackbirds Ageliaus phoeniceus respond differently to song types with different performance levels. Journal of Avian Biology. 38(1):122-127.

Derryberry EP, Seddon N, Claramunt S, Tobias JA, Baker A, Aleixo A, Brumfield RT. 2012. Correlated evolution of beak morphology and song in the neotropical woodcreeper radiation. Evolution: International Journal of Organic Evolution. 66(9):2784-2797.

Drăgănoiu TI, Nagle L, Kreutzer M. 2002. Directional female preference for an exaggerated male trait in canary (Serinus canaria) song. Proceedings of the Royal Society of London B: Biological Sciences. 269(1509):2525-2531.

DuBois AL, Nowicki S, Searcy WA. 2009. Swamp sparrows modulate vocal performance in an aggressive context. Biology letters. 5(2):163-165.

DuBois AL, Nowicki S, Searcy WA. 2011. Discrimination of vocal performance by male swamp sparrows. Behav Ecol Sociobiol. 65(4):717-726.

Forstmeier W, Kempenaers B, Meyer A, Leisler B. 2002. A novel song parameter correlates with extra-pair paternity and reflects male longevity. Proceedings of the Royal Society of London B: Biological Sciences. 269(1499):1479-1485.

Geberzahn N, Aubin T. 2014a. Assessing vocal performance in complex birdsong: A novel approach. BMC Biology. 12:1-9.

Geberzahn N, Aubin T. 2014b. How a songbird with a continuous singing style modulates its song when territorially challenged. Behav Ecol Sociobiol. 68(1):1-12.

Geraci M. 2014. Linear quantile mixed models: the lqmm package for Laplace quantile regression. Journal of Statistical Software. 57(13):1-29.

Guilford T, Dawkins MS. 1991. Receiver psychology and the evolution of animal signals. Animal Behaviour. 42(1):1-14.

Hartley RS, Suthers RA. 1989. Airflow and pressure during canary song: direct evidence for mini-breaths. Journal of Comparative Physiology A. 165(1):15-26.

Hedley RW, Logue DM, Benedict L, Mennill DJ. 2018. Assessing the similarity of song-type transitions among birds: evidence for interspecies variation. Animal Behaviour. 140:161production: experimental manipulation of beak movements. Journal of Experimental Biology. 203(12):1845-1855. 
642 Holveck M-J, Riebel K. 2007. Preferred songs predict preferred males: consistency and repeatability of zebra finch females across three test contexts. Animal Behaviour. 74(2):297-309.

Illes AE, Hall ML, Vehrencamp SL. 2006. Vocal performance influences male receiver response

Kaluthota CD, Medina OJ, Logue DM. 2019. Quantifying song categories in Adelaide's Warbler (Setophaga adelaidae). Journal of Ornithology.1-11.

Kroodsma D. 2017a. Birdsong 'performance'studies: A sad commentary. Animal Behaviour. 133:209-210.

Kroodsma D. 2017b. Birdsong performance studies: a contrary view. Animal Behaviour. 125:e1e16.

654 mLachlan R. 2007. Luscinia: a bioacoustics analysis computer program.

655 Larimer JL, Dudley R. 1995. Accelerational implications of hummingbird display dives. The Auk. 112(4):1064-1066.

Leadbeater E, Goller F, Riebel K. 2005. Unusual phonation, covarying song characteristics and song preferences in female zebra finches. Animal Behaviour. 70(4):909-919.

Logue DM, Forstmeier W. 2008. Constrained performance in a communication network: implications for the function of song-type matching and for the evolution of multiple ornaments. The American naturalist. 172(1):34-41.

662 Moseley DL, Lahti DC, Podos J. 2013. Responses to song playback vary with the vocal performance of both signal senders and receivers. Proceedings of the Royal Society B: Biological Sciences. 280(1768):20131401.

665 Mota PG, Cardoso GC. 2001. Song organisation and patterns of variation in the serin (Serinus serinus). Acta Ethologica. 3(2):141-150.

Murai M, Backwell PR. 2006. A conspicuous courtship signal in the fiddler crab Uca perplexa: female choice based on display structure. Behav Ecol Sociobiol. 60(5):736-741.

Nowicki S, Westneat M, Hoese W. 1992. Birdsong: motor function and the evolution of

671 Oberweger K, Goller F. 2001. The metabolic cost of birdsong production. Journal of Experimental Biology. 204(19):3379-3388. 
673 Phillips JN, Derryberry EP. 2017a. Equivalent effects of bandwidth and trill rate: support for a

674

675

676

677

678

679

680

681

682

683

684

685

686

687

688

performance constraint as a competitive signal. Animal behaviour. 132:209-215.

Phillips JN, Derryberry EP. 2017b. Vocal performance is a salient signal for male-male

competition in White-crowned Sparrows. The Auk: Ornithological Advances. 134(3):564-574.

Plummer EM, Goller F. 2008. Singing with reduced air sac volume causes uniform decrease in airflow and sound amplitude in the zebra finch. Journal of Experimental Biology. 211(1):66-78.

Podos J. 1997. A performance constraint on the evolution of trilled vocalizations in a songbird family (Passeriformes: Emberizidae). Evolution; international journal of organic evolution. 51(2):537-551.

Podos J. 2001. Correlated evolution of morphology and vocal signal structure in Darwin's finches. Nature. 409:185-188.

Podos J. 2017. Birdsong performance studies: reports of their death have been greatly exaggerated. Animal Behaviour. 125:e17-e24.

Podos J, Lahti DC, Moseley DL. 2009. Vocal performance and sensorimotor learning in songbirds. Advances in the Study of Behavior. 40:159-195.

Podos J, Moseley DL, Goodwin SE, McClure J, Taft BN, Strauss AV, Rega-Brodsky C, Lahti DC. 2016. A fine-scale, broadly applicable index of vocal performance: frequency excursion. Animal behaviour. 116:203-212.

Podos J, Nowicki S. 2004. Beaks, adaptation, and vocal evolution in Darwin's finches. AIBS Bulletin. 54(6):501-510.

mRudis B, Bolker B, Schulz J, Kothari A, Sidi J. 2017. ggalt: extra coordinate systems, ‘Geoms’, statistical transformations, scales and fonts for 'ggplot2'.

Schraft HA, Medina OJ, McClure J, Pereira DA, Logue DM. 2017. Within-day improvement in a behavioural display: wild birds 'warm up'. Animal Behaviour. 124:167-174.

Smith JM, Harper D. 2003. Animal signals. Oxford University Press.

Staicer C. 1991. The role of male song in the socioecology of the tropical resident Adelaide's warbler (Dendroica adelaidae). University of Massachusetts. 
Stoffel MA, Nakagawa S, Schielzeth H. 2017. rptR: Repeatability estimation and variance decomposition by generalized linear mixed-effects models. Methods in Ecology and Evolution. 8(11):1639-1644.

Suthers RA. 2004. How birds sing and why it matters. Nature's music: the science of birdsong Elsevier Academic Press, San Diego.272-295.

Suthers RA, Zollinger SA. 2004. Producing song: the vocal apparatus. Annals of the New York Academy of Sciences. 1016(1):109-129.

Team R. 2015. RStudio: Integrated Development for R.

Toms JD. 2010. Adelaide's Warbler (Setophaga adelaidae). version 1.0. Ithaca, New York, USA: Cornell Lab of Ornithology; [accessed 2018 July 4].

Vehrencamp SL, Yantachka J, Hall ML, de Kort SR. 2013. Trill performance components vary with age, season, and motivation in the banded wren. Behav Ecol Sociobiol. 67(3):409419.

Westneat MW, Long J, Hoese W, Nowicki S. 1993. Kinematics of birdsong: functional correlation of cranial movements and acoustic features in sparrows. Journal of Experimental Biology. 182(1):147-171.

Wickham H, Chang W. 2008. ggplot2: An implementation of the Grammar of Graphics. R package version 07, URL: http://CRAN R-project org/package= ggplot2.

Wilson DR, Bitton PP, Podos J, Mennill DJ. 2014. Uneven sampling and the analysis of vocal performance constraints. The American naturalist. 183(2):214-228. 


\section{Tables and table legends}

725

726 Table 1. Sample sizes, separated by individual.

\begin{tabular}{llll} 
individual & songs & notes & song types \\
\hline DDLb & 605 & 13,465 & 19 \\
KYK & 110 & 2,449 & 23 \\
LgRLg & 255 & 6,427 & 17 \\
LgWV & 254 & 5,955 & 16 \\
OWO & 461 & 11,512 & 18 \\
PDP & 139 & 3,064 & 23 \\
RbRbO & 425 & 9,137 & 24 \\
RDY & 509 & 13,895 & 27 \\
ROLb & 121 & 2,698 & 20 \\
Total & $\mathbf{2 , 8 7 9}$ & $\mathbf{6 8 , 6 0 2}$ & $\mathbf{1 8 7} *$ \\
\hline
\end{tabular}

$727 *$ There were 61 unique song types. 
729 Table 2. Intra-class correlation and skewness values for note-level deviation scores.

\begin{tabular}{llll} 
statistic (sample) & note length vs. gap & note BW vs. & gap BW vs. \\
& length & note length & gap length \\
\hline individual ICC (all & $\mathrm{r}=0.178$ & $\mathrm{r}=0.161$ & $\mathrm{r}=0.206$ \\
songs) & $\mathrm{p}<0.0001$ & $\mathrm{p}<0.0001$ & $\mathrm{p}<0.0001$ \\
individual ICC (song & $\mathrm{r}=0.154$ & $\mathrm{r}=0.056$ & $\mathrm{r}=0.116$ \\
types w/in individual) & $\mathrm{p}=0.0002$ & $\mathrm{p}=0.052$ & $\mathrm{p}=0.0025$ \\
song type ICC (song & $\mathrm{r}=0.167$ & $\mathrm{r}=0.641$ & $\mathrm{r}=0.372$ \\
types w/in individual) & $\mathrm{p}=0.0098$ & $\mathrm{p}<0.0001$ & $\mathrm{p}<0.0001$ \\
note-level skewness & $\mathrm{sk}=3.28$ & & $\mathrm{sk}=2.21$ \\
(notes) & & $\mathrm{sk}=1.29$ & \\
avg. note-level & $\mathrm{sk}=0.58$ & & $\mathrm{sk}=0.51$ \\
skewness (songs) & & $\mathrm{sk}=0.55$ &
\end{tabular}

ICCs rely on note-level deviation scores averaged over songs. 
733 Table 3. Results of intra-class correlation analysis and skewness analysis on song-level deviation

734 scores and FEX.

statistic (sample) TR vs. length vs. length vs. TR vs. FEX

mean TR PoS PoS

BW

\begin{tabular}{|c|c|c|c|c|c|}
\hline & $\mathrm{r}=0.161$ & $\mathrm{r}=0.158$ & $\mathrm{r}=0.156$ & $r=0.144$ & $\mathrm{r}=0.140$ \\
\hline individual ICC (all & $\mathrm{p}<$ & $\mathrm{p}<$ & $\mathrm{p}<0.0001$ & $\mathrm{p}<0.0001$ & $\mathrm{p}<0.0001$ \\
\hline songs) & 0.0001 & 0.0001 & & & \\
\hline individual ICC (song & $r=0.009$ & $\mathrm{r}=0.079$ & $\mathrm{r}=0.126$ & $\mathrm{r}=0.148$ & $r=0.068$ \\
\hline types w/in & $\mathrm{p}=0.455$ & $\mathrm{p}=0.011$ & $\mathrm{p}=0.0006$ & $\mathrm{p}=0.0002$ & $\mathrm{p}=0.040$ \\
\hline \multicolumn{6}{|l|}{ individual) } \\
\hline song type ICC (song & $r=0.556$ & $r=0.604$ & $\mathrm{r}=0.280$ & $\mathrm{r}=0.117$ & $r=0.534$ \\
\hline types w/in & $\mathrm{p}<$ & $\mathrm{p}<$ & $\mathrm{p}<0.0001$ & $\mathrm{p}=0.0254$ & $\mathrm{p}<0.0001$ \\
\hline individual) & 0.0001 & 0.0001 & & & \\
\hline skewness (all songs) & -0.092 & -0.264 & 0.162 & 0.138 & -0.070 \\
\hline
\end{tabular}


737 Table 4. Pearson's correlation matrix for song-level deviation scores, frequency excursion, and 738 note-level deviation scores averaged over songs.

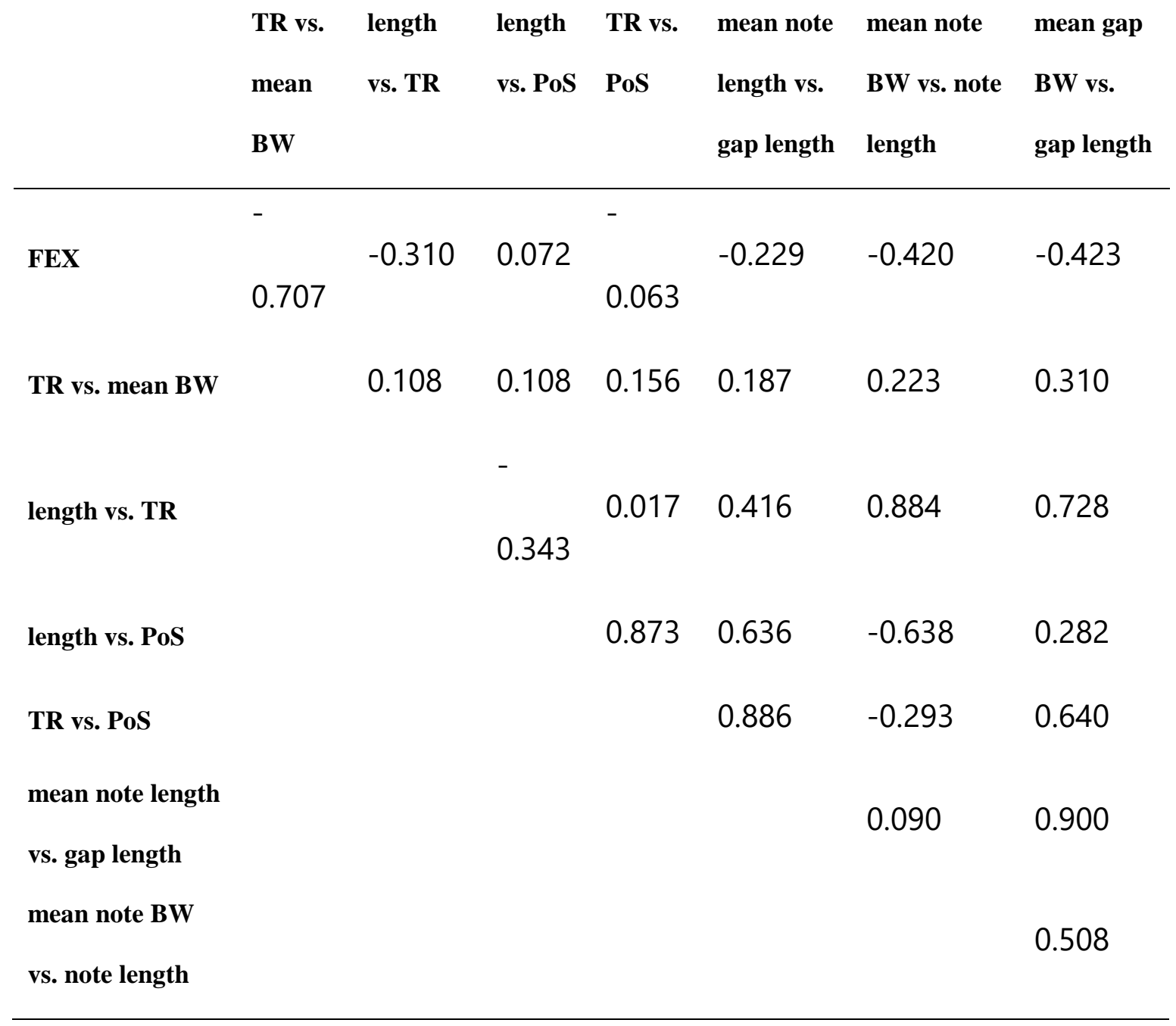


bioRxiv preprint doi: https://doi.org/10.1101/664896; this version posted June 11, 2019. The copyright holder for this preprint (which was not certified by peer review) is the author/funder, who has granted bioRxiv a license to display the preprint in perpetuity. It is made available under aCC-BY-NC-ND 4.0 International license.

\section{$741 \quad$ Figures}

\section{Figure 1}

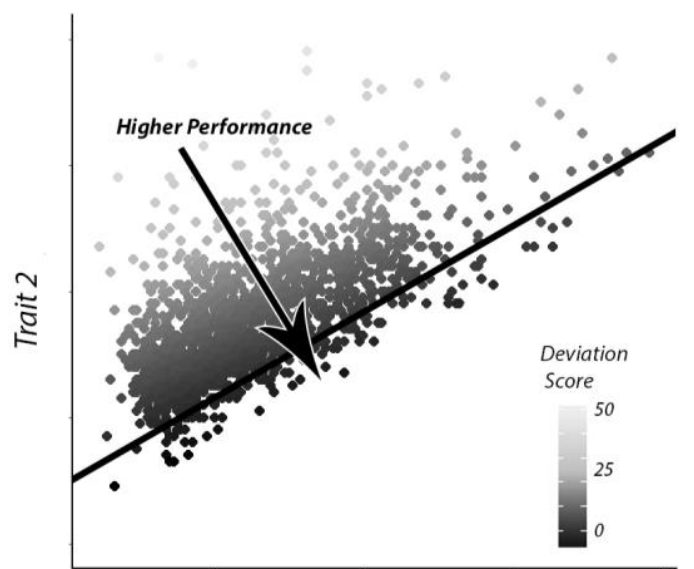

Trait 1 
bioRxiv preprint doi: https://doi.org/10.1101/664896; this version posted June 11, 2019. The copyright holder for this preprint (which was not certified by peer review) is the author/funder, who has granted bioRxiv a license to display the preprint in perpetuity. It is made available under aCC-BY-NC-ND 4.0 International license.

\section{Figure 2}

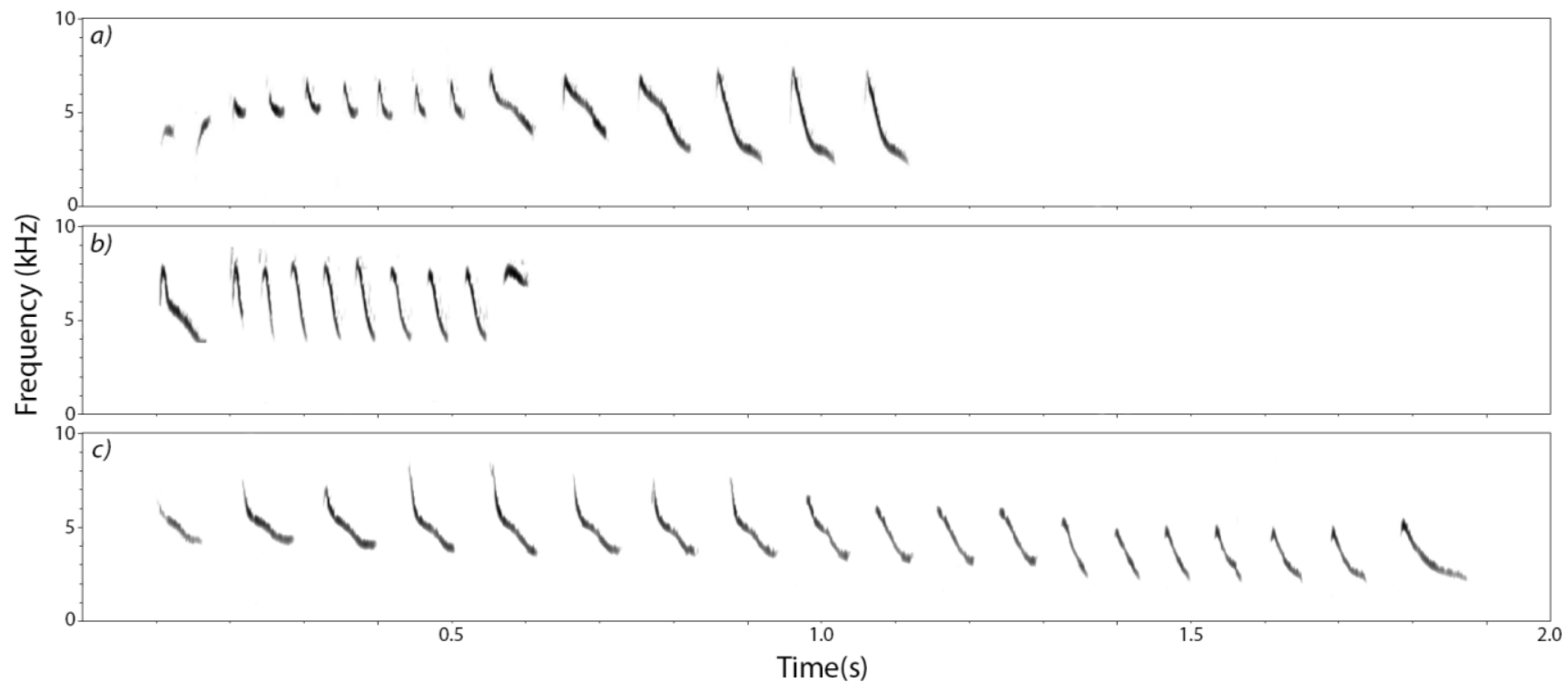

747 
bioRxiv preprint doi: https://doi.org/10.1101/664896; this version posted June 11 2019. The copyright holder for this preprint (which was not certified by peer review) is the author/funder, who has granted bioRxiv a license to display the preprint in perpetuity. It is made available under aCC-BY-NC-ND 4.0 International license.

Figure 3

749

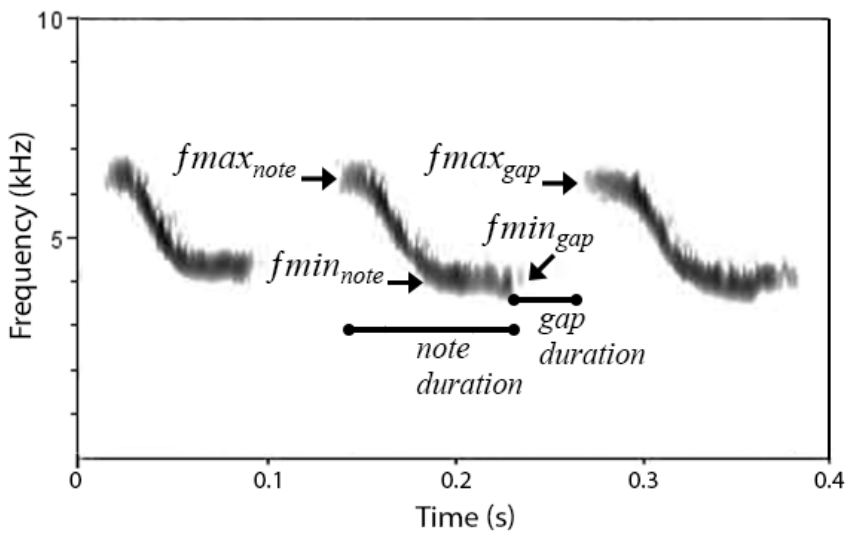

750 
Figure 4
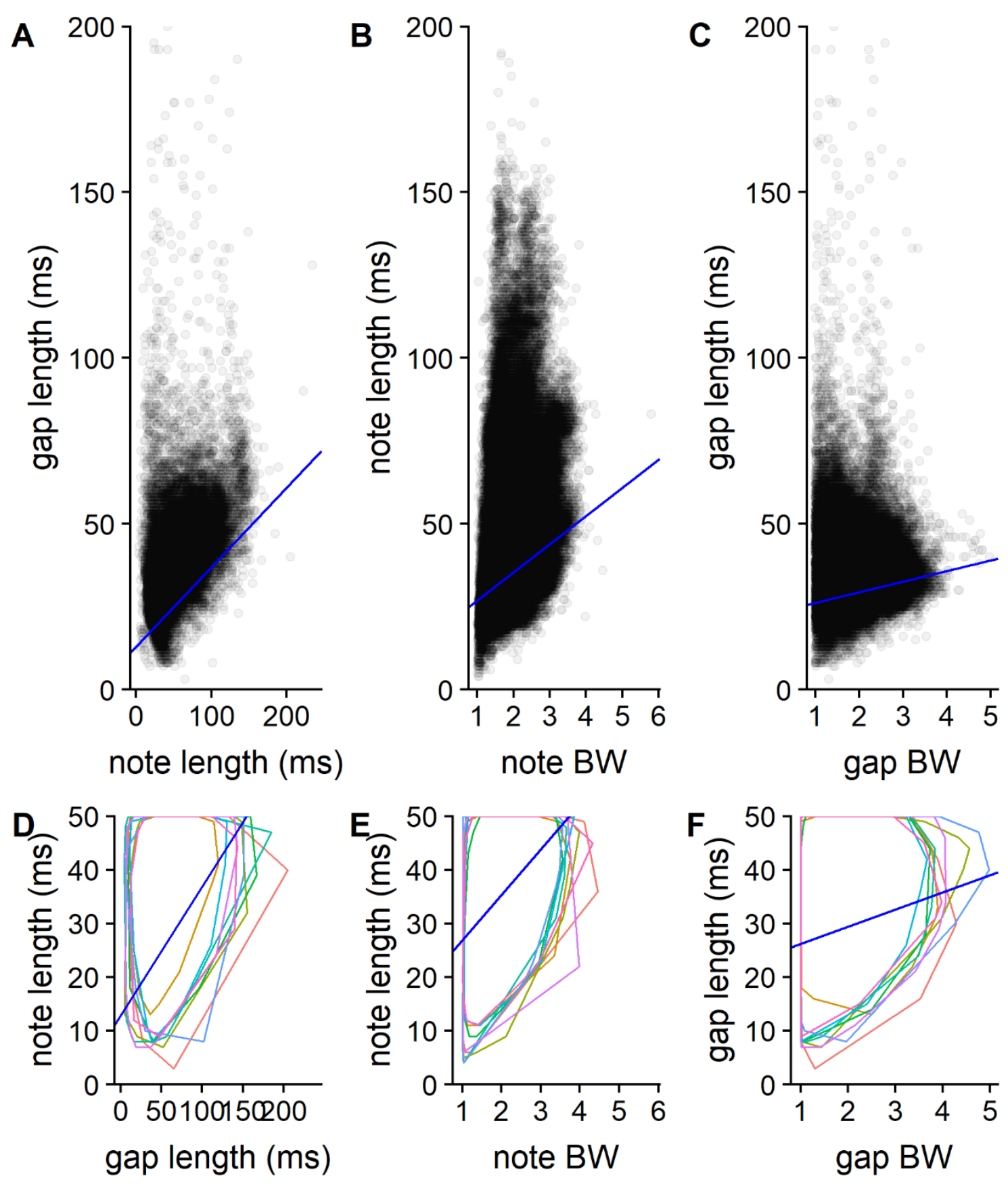
Figure 5
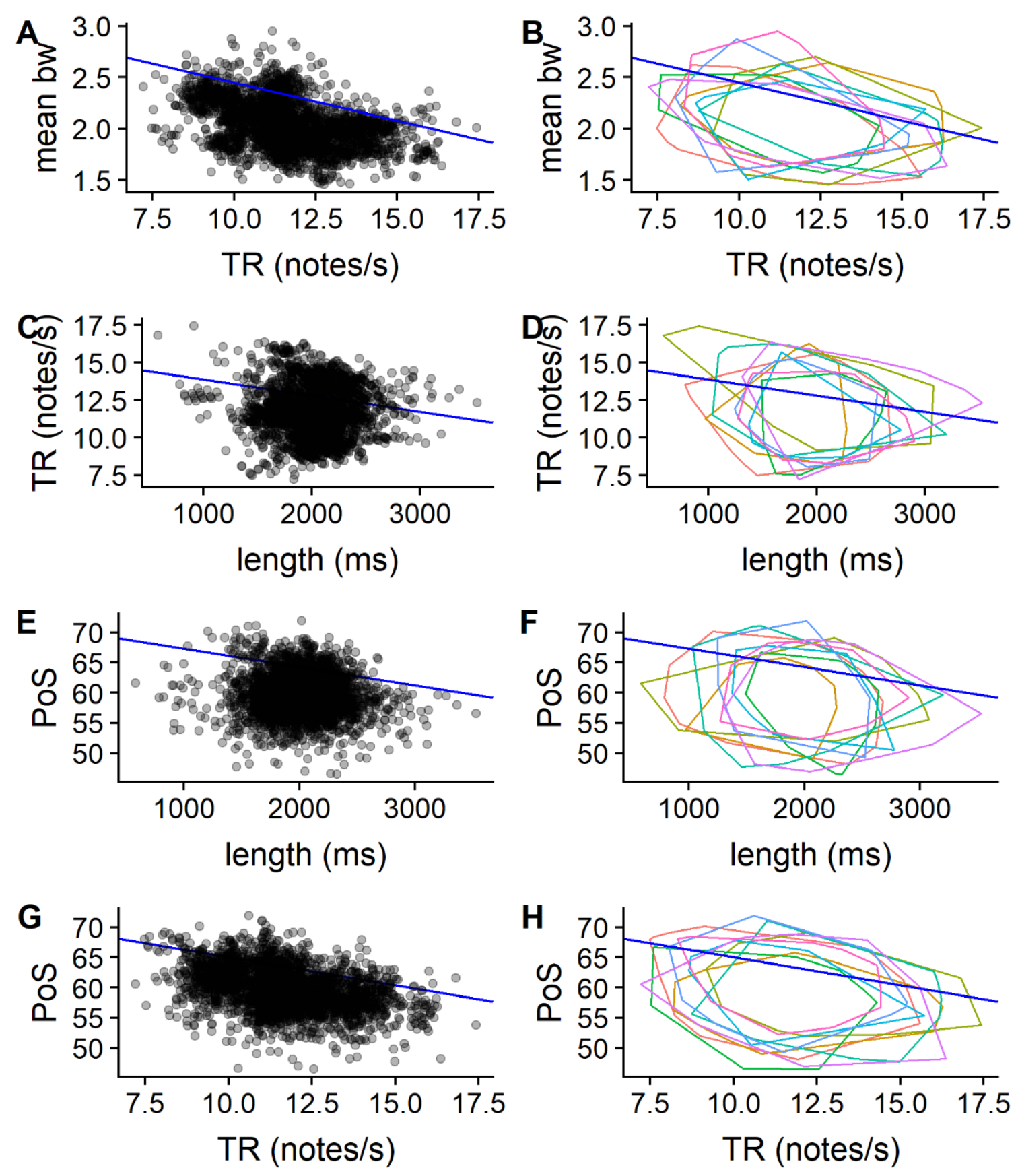


\section{Figure captions}

759 Figure 1: Scatterplot depicting a hypothetical performance constraint between two traits. The

$76010^{\text {th }}$ quantile regression line is shown in black. For comparisons in which the trait on the $\mathrm{x}$-axis

761 scales positively with performance and the trait on the y-axis scales negatively with performance,

762 any performance limit would follow the lower edge of the distribution and slope upward, and

763 performance would increase as one moves down and to the right (this figure, Fig. 4). For

764 comparisons in which both the $\mathrm{x}$ - and $\mathrm{y}$-axes scale positively with performance, performance

765 limits follow the upper edge of the distribution and slope downward, and performance increases

766 as one moves up and to the right (e.g., Fig. 5).

767 Figure 2: Sound spectrograms of three Adelaide’s warbler song types.

768 Figure 3. Six note-level measurements. The focal note is in the middle. We used Fmax note $_{\text {and }}$

$769 F_{\text {Fin }}$ note to determine the note frequency bandwidth, and $F_{\max _{\text {gap }}}$ and $F \min _{\text {gap }}$ to determine the

770 gap frequency bandwidth.

771 Figure 4: Plots comparing acoustic properties of Adelaide's warbler notes. Data points in

772 scatterplots (A-C) are semi-transparent to show density. Polygon plots (D- F) focus on the

773 bottom of each distribution. Each colored polygon represents the limits of an individual males'

774 note sample. Royal blue lines represent $10^{\text {th }}$ quantile regression lines. Scales differ between the

775 scatterplots and the polygon plots.

776 Figure 5: Plots comparing acoustic properties of Adelaide's warbler songs. Data points in

777 scatterplots (A, C, E, G) are semi-transparent to facilitate interpretation. Each colored polygon in

778 the polygon plots (B, D, F, H) represents the limits of an individual males' song sample. Royal

779 blue lines represent $90^{\text {th }}$ quantile regression lines. 